nant les deux points $\lambda_{0}$ et $\lambda^{\prime}$. Cette équation étant d'ordre $n-1$, on a, par l'hypothèse admise, $x(\lambda)=0$ pour $\lambda \in I$ et en particulier $x\left(\lambda^{\prime}\right)=0$.

$2^{0}$ On a dans un entourage de $\lambda_{0}$

$$
a_{m} x^{(m)}(x)+\ldots \dot{\dagger} a_{0} x(x)=0 .
$$

Soit alors $\left(a^{\prime}, \beta^{\prime}\right)$ le plus grand des tels entourages. Comme l'ordre de (3) est $m \leqslant n-1$, on a identiquement $x(x)=0$ dans $\left(\alpha^{\prime}, \beta^{\prime}\right)$. Lorsque $\lambda^{\prime}$ est en dehors de $\left(\alpha^{\prime}, \beta^{\prime}\right)$, on pent fixer $x$ et $\lambda_{1}$ de manière que

et

$$
\lambda_{1} \epsilon\left(\alpha^{\prime}, \beta^{\prime}\right), \quad\left|\chi-\lambda_{1}\right| \leqslant \min \left(\lambda^{\prime}-\alpha, \beta-\lambda^{\prime}\right)
$$

$$
a_{m} x^{(m)}(x)+\ldots+a_{0} x(x) \neq 0,
$$

ce qui réduit le raisonnement au cas précédent.

(Reçu par la Rédaction le 17. 5. 1949).

\section{Sur les fondements du calcul opératoire}

\section{JAN G.-MIKUSINSKI (Wroclaw).}

\section{Introduction.}

On connaît les méthodes suivantes d'introduire le calcul opératoire:

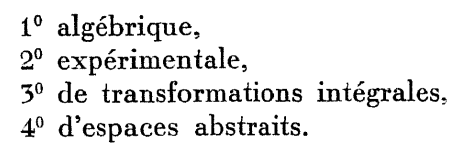

$1^{0}$ algébrique,

$2^{\circ}$ expérimentale,

$3^{0}$ de transformations intégrales,

$4^{0}$ d'espaces abstraits.

La méthode algébrique, employée déjà par $\mathrm{L}_{\mathrm{AGRANGE}}{ }^{1}$ ), peut être caractérisée aisément en termes de l'algèbre abstraite. On parvient, par l'adjonction de l'opérateur différentiel $s$ au corps des nombres complexes, à un corps des opérateurs qui sont ensuite appliqués aux éléments d'un anneau $C$ de fonctions, où l'addition garde son sens habituel et la multiplication est définie par le produit de composition.

Le défaut principal de cette méthode est l'impossibilité d'introduire les opérateurs de type $e^{-s \lambda}$ et $e^{-V^{-} \lambda}$ qui sont indispensables pour des applications aux équations aux dérivées partielles.

La méthode expérimentale (de $\mathrm{H}_{\mathrm{AEviside}}{ }^{2}$ ) traite les calculs avec les opérateurs comme ceux avec les nombres ou les fonctions: on écrit par exemple

$$
e^{-s \lambda}=1-\frac{s \lambda}{1 !}+\frac{s^{2} \lambda^{2}}{2 !}-\ldots
$$

1) Voir K. T. Vahlen [24]. Les numéros entre crochets indiquent les publications dont la liste se trouve à la fin de ce mémoire (p. 69-70).

${ }^{2}$ O. Heaviside [13]. 
sans se soucier de la convergence de cette série. La seule raison d'employer cette méthode était qu'elle conduisait, en général, aux résultats corrects, susceptibles d'être vérifiés ensuite par une autre voie.

Depuis l'apparition des travaux de Carson et de Dortsch ${ }^{3}$ ) les méthodes précédentes ont été remplacées presque entièrement par celles de transformations intégrales. Tous les traités modernes, et dont le nombre augmente chaque année, suivent cette voie $\left.{ }^{4}\right)$. La propagation rapide de la nouvelle méthode peut s'expliquer par la commodité avec laquelle elle permet de résoudre non seulement les équations différentielles ordinaires, mais aussi celles aux dérivées partielles, même dans les cas où les deux méthodes précédentes étaient impuissantes.

Un certain défaut de la méthode de transformations intégrales consiste en ce qu'elle rétrécit inutilement le champ des applications à des fonctions $f(\boldsymbol{t})$, pour lesquelles l'intégrale de Laplace $\int_{0}^{\infty} e^{-s t} f(t) d t$ converge; elle ne fournit done pas, sans un raisonnement accessoire, la solution de-l'équation suivante par exemple:

$$
\frac{d}{d t} x(t)+x(t)=(2 t-1) e^{t^{2}}
$$

Cette restriction est génante aussi dans le cas d'équations aux dérivées partielles, où elle empêche en particulier d'assurer l'unicité des solutions obtenues (voir \$ 25), sinon en restreignant la classe des fonctions admissibles. En outre, il est peu commode, au point de vue pratique, d'opérer simultanément avec deux classes de fonctions, ce qui nécessite une double notation.

La méthode des espaces abstraits a été proposée tout récemment par l'auteur ${ }^{5}$ ). On y considère l'anneau $C$ de fonctions

3) J. Carson [3], G. Doetsch [8]

1) Mentionnons-en les auteurs suivants: Cars on [3] (1926), Jeff reys [15] (1931), Humbert [14] (1934), Dahr [6] (1935), Berg [1] (1936), Doetsch [8] Efros et Danilevskii [12] (1937), Lurie [16] (1938), M c Lachlan [17] (1939). Wagner [25] (1940), Carslaw and Jaeger [2] (1941), Churchill [4] (1944), Ditkin [7] (1947), Mc Lachlan [18] (1948). Cette liste ne contient que les nuels ou les monographies et ne prétend pas à être complète.

5) J. G. -Mikusiński [21]. où l'on introduit ensuite une topologie faible. En le complétant par le procédé de CANTOR, on l'enrichit des éléments nouveaux qui possèdent les propriétés des opérateurs de Heaviside. Cette méthode attribue donc aux opérateurs un sens direct et n'introduit pas de restrictions superflues concernant la classe des fonctions considérées.

Ce mémoire a pour but de montrer comment la méthode algébrique, convenablement complétée, peut servir au calcul opératoire de fondement à la fois complet et libre de restrictions inutiles. Grâce à un théorème de Trtchmarsh ${ }^{6}$ ), cette méthode peut être rendue symétrique et élégante: elle fournit d'un seul coup, en immergeant l'anneau $C$ dans un corps, tous les opérateurs de Heaviside. On n'aura plus besoin de traiter séparément le corps d'opérateurs et l'anneau de fonctions, car tous les deux feront parties du même corps. En introduisant ensuite la notion de convergence d'une manière convenable, le calcul devient applicable aux équations aux dérivées partielles et permet non seulement de les résoudre, mais aussi de démontrer l'unicité de leurs solutions ${ }^{\top}$.

\section{Algèbre des opérateurs.}

\$1. Le corps des opérateurs. Désignons par $C$ l'ensemble des fonctions complexes continues $a=\{a(t)\}^{8}$ ) de variable réelle $t$, définies pour $t \geqslant 0$. Définissons dans $C$ l'addition et la multiplication en posant:

$$
\begin{aligned}
& a+b=\{a(t)\}+\{b(t)\}=\{a(t)+b(t)\}, \\
& a \cdot b=\{a(t)\} \cdot\{b(t)\}=\left\{\int_{0}^{t} a(t-\tau) b(\tau) d \tau\right\} .
\end{aligned}
$$

Cela posé, on vérifie facilement que $C$ est un anneau commutatif, sans unité. D'après un théorème de Trтchmarsh ${ }^{9}$ ) sur le produit de composition $\int_{0}^{t} a(t-\tau) b(\tau) d \tau$, l'anneau n'a pas de

9) E. C. Titchmarsh [22] et [23]; voir aussi J. Dufresnoy [10] et M. Crum [5].

7) Pour l'unicité voir aussi S. Drobot et J. G.-Mikusiński [9].

8) Nous convenons de désigner par a ou par $\{a(t)\}$ les fonctions, en réservant le symbole a $(t)$ à leur valeur au point $t$

9) E. C. Titchmarsh [22], p. 286, Theorem VII 
diviseurs de zéro, c'est-à-dire que $a b=0$ entraîne $a=0$ ou $b=0$. Il s'ensuit que l'anneau $C$ peut être immergé dans un corps $A$.

En pratique, on considère comme éléments de $A$ les fractions $\stackrel{a}{b}(b \neq 0)$ et on pose par définition:

$$
\frac{a}{b}=\frac{c}{d} \text { lorsque } a d=b c,
$$

$a=\frac{a k}{k}, k$ étant un élément non nul, fixé arbitrairement.

$A$ est alors le moindre corps commutatif dont le sousensemble composé d'éléments de la forme $\frac{a k}{k}$ est isomorphe à $C$.

Il importe de remarquer que ce corps et cette isomorphic sont déterminés univoquement quel que soit l'élément choisi $k$. car on a toujours $\frac{a k^{\prime}}{k^{\prime}}=\frac{a k^{\prime \prime}}{k^{\prime \prime}}$ pour $k^{\prime}$ et $k^{\prime \prime}$ non nuls. En posant $a=\frac{a k}{k}$, nous pouvons donc toujours identifier la fonction a avec tout élément de forme $\frac{a k}{k}$, ce que nous ferons partout dans la suite.

L'addition et la multiplication dans $A$ sont définies en posant:

$$
\begin{gathered}
\frac{a}{b}+\frac{c}{d}=\frac{a d+b c}{b d}, \\
\frac{a}{b} \cdot \frac{c}{d}=\frac{a c}{b d} .
\end{gathered}
$$

Cela posé, les éléments du corps $A$ seront dits opérateurs. Pour désigner les opérateurs, nous emploierons dans la suitc les lettres latines $a, b, \ldots$ aussi bien que pour désigner les fonctions; il faut d'ailleurs considérer les fonctions appartenant. à $C$ comme des opérateurs particuliers.

\$ 2. Opérateur intégral. Nous désignerons par $l=\{1\}$ la fonction constante dont la valeur est égale à 1 pour tout $t \geqslant 0$. On voit d'après (1) que, a étant une fonction appartenant à $C$, on a

$$
l a=\left\{\int_{0}^{t} a(\tau) d \tau\right\}
$$

la multiplication d'une fonction $a=\{a(t)\}$ par $l$ est donc l'intégration de cette fonction entre les limites 0 et $t$. C'est pourquoi nous appellerons $l$ l'opérateur intégral.

§ 3. Fonctions sommables. Soit $£$ la classe des opérateurs a pour lesquels le produit la est une fonction qui se laisse représenter dans la forme

$$
l a=\left\{\int_{0}^{t} a(\tau) d \tau\right\}
$$

où $\{a(t)\}$ est une fonction sommable (au sens ordinaire) dans tout intervalle $0 \leqslant t \leqslant t_{0}$.

La fonction $\{a(t)\}$ qui correspond ainsi à l'opérateur a est déterminée à un ensemble de mesure nulle près. Réciproquement, à toute fonction $\{a(t)\}$, sommable dans tout intervalle $0 \leqslant t \leqslant t_{0}$, vient correspondre univoquement un opérateur de la classe $t$.

La classe $L$ des fonctions sommables dans tout intervalle $0 \leqslant t \leqslant t_{0}$ est isomorphe à la classe $t$ des opérateurs, pourvu que l'addition et la multiplication dans $L$ soient aussi entendues au sens (1). En effet, le produit de composition de deux fonctions de la classe $L$ est encore une fonction de cette classe et il obéit aux lois de commutativité et d'associativité ${ }^{10}$ ). Si maintenant

$$
l a=\left\{\int_{0}^{t} a(\tau) d \tau\right\}=\{A(t)\} \quad \text { et } \quad l b=\left\{\int_{0}^{t} b(\tau) d \tau\right\}=\{B(t)\},
$$

on a

$$
l^{2} a b=l a \cdot l b=\left\{\int_{0}^{t} A(t-\tau) B(\tau) d \tau\right\}=l\left\{\int_{0}^{t} C(\tau) d \tau\right\}
$$

où $C(t)=\int_{0}^{t} a(t-\tau) b(\tau) d \tau$. Il résulte de $(\mathfrak{3})$ que.

$$
l a b=\left\{\int_{0}^{t} C(\tau) d \tau\right\},
$$

d'où l'isomorphie par rapport à la multiplication. Pour l'addition, l'isomorphie est triviale.

L'isomorphie des classes $L$. et $\ell$ étant ainsi établie, on peut identifier les opérateurs a de $\ell$ avec les fonctions $\{a(t)\}$ de $L$

10) J. G. - Mikusiński [19], p. 9-11. 
et écrire tout simplement $a=\{a(t)\}$, ce qui est commode dans les calculs pratiques.

\$ 4. Opérateurs numériques. Soit $a$ un nombre complexe quelconque; posons

$$
[a]=\frac{\{a\}}{l},
$$

où $\{a\}$ est la fonction constante de valeur $a$ pour tout $t \geqslant 0$. On vérifie aussitôt les égalités

$$
[\alpha]+[\beta]=[\alpha+\beta] \text { et }[\alpha][\beta]=[\alpha \beta] .
$$

Ces égalités expriment l'isomorphie entre l'ensemble des éléments $[a]$ et celui des nombres complexes; on appellera donc ces éléments opérateurs numériques ou nombres tout court, et l'on écrira

$$
[a]=\alpha
$$

On a donc la formule

$$
a=\frac{\{a\}}{l}
$$

Ainsi le corps $A$ des opérateurs peut être considéré comme une extension du corps des nombres complexes. On aura donc en particuliex:

$$
a+0=a, \quad 0 a=0 \quad \text { et } \quad 1 a=a
$$

pour tout. opérateur $a$, c'est-à-dire que les nombres' 0 et 1 sont respectivement le zéro et l'unité du corps des opérateurs.

Il faut, par contre, distinguer les opérateurs numériques $a$ des fonctions constantes $\{\alpha\}$, pour lesquelles les lois d'addition et de multiplication s'expriment, en vertu de (1), par les formules

$$
\{\alpha\}+\{\beta\}=\{\alpha+\beta\} \quad \text { et } \quad\{\alpha\}\{\beta\}=\{\alpha \beta t\}
$$

On a

$$
a\{a(t)\}=\{a a(t)\}
$$

pour toute fonction $\{a(t)\}$ continue ou sommable. En effet, on a d'après (4) et (2)

$$
a\{a(t)\}=\frac{\{a\}\{a(t)\}}{l}=\frac{1}{l}\left\{\int_{0}^{t} a a(\tau) d \tau\right\}=\frac{1}{l} \cdot l\{a a(t)\}=\{a a(t)\} .
$$

La formule (5) exprime une règle pratique qui permet d'inclure le nombre $\alpha$ dans \{\} . On a en particulier

et pour $\beta=1$

$$
\alpha\{\beta\}=\{\alpha \beta\}
$$

$$
\alpha 1=\{\alpha\} .
$$

C'est ainsi, par exemple, que

$$
2 \cdot 3=6, \quad 2\{3\}=\{6\}, \quad\{2\}\{3\}=\{6 t\} .
$$

Notons enfin la formule générale

(6) $\quad(\alpha+\{a(t)\})(\beta+\{b(t)\})=\alpha \beta+\left\{\alpha b(t)+\beta a(t)+\int_{0}^{t} a(t-\tau) b(\tau) d \tau\right\}$.

§ 5. L'opérateur différentiel. Posons par définition

$$
s=\frac{1}{l}
$$

$s$ est donc l'inverse de $l$.

Si a est une fonction absolument continue pour $t \geqslant 0$, on a (7)

$$
s a=a_{0}+a^{\prime},
$$

où $a_{0}$ est l'opérateur numérique correspondant à la valeur de a au point $t=0$ et $a^{\prime}$ est la dérivée de a.

On a en effet

$$
\{a(t)\}=\{a(0)\}+\left\{\int_{0}^{t} a^{\prime}(\tau) d \tau\right\}
$$

c'est-à-dire $\{a(t)\}=l a_{0}+l\left\{a^{\prime}(t)\right\}$, et en multipliant cette égalité par $s$, il vient (z).

En particulier si $a_{0}=0$, la formule (7) devient

$$
s a=a^{\prime}
$$

dans ce cas, la multiplication d'une fonction par $s$ est donc la différentiation de cette fonction, ce qui justifie la dénomination opérateur différentiel, assignée à $s$.

Si la fonction a possède la $(n-1)$-ème dérivée absolument continue, on déduit de (7) par induction que

$$
s^{n} a=s^{n-1} a_{0}+\ldots+s a_{0}^{(n-2)}+a_{0}{ }^{(n-1)}+a^{(n)},
$$

où $a_{0}^{(i)}$ est l'opérateur numérique égal à la valeur de la $i$-ème dérivée de $a$ au point $t=0$ et $a^{(n)}$ est la $n$-ième dérivée de $a$. 
Pour les applications aux équations différentielles, il est commode d'avoir la dernière formule dans la forme

$$
a^{(n)}=s^{n} a-s^{n-1} a_{0}-\ldots-s a_{0}^{(n-2)}-a_{0}{ }^{(n-1)} .
$$

\$ 6. Opérateurs rationnels en s. On a

$$
\frac{1}{(s-a)^{n}}=\left\{\frac{t^{n-1}}{(n-1) !} e^{\alpha t}\right\} \quad \text { pour } n=1,2, \ldots
$$

En effet, il vient d'après les formules (7) et (5)

$$
(s-\alpha)\left\{e^{\alpha t}\right\}=s\left\{e^{\alpha t}\right\}-\alpha\left\{e^{\alpha t}\right\}=\left(1+\left\{\alpha e^{\alpha t}\right\}\right)-\left\{\alpha e^{\alpha t}\right\}=1,
$$

ce qui entraîne (9) pour $n=1$. En admettant maintenant que la formule (9) est vraie pour un certain $n$, on trouve qu'elle l'est pour $n+1$, car

$$
(s-\alpha)\left\{\frac{t^{n}}{n !} e^{\alpha t}\right\}=s\left\{\frac{t^{n}}{n !} e^{\alpha t}\right\}-\alpha\left\{\frac{t^{n}}{n !} e^{\alpha t}\right\}=\left\{\frac{t^{n-1}}{(n-1) !} e^{\alpha t}\right\}=\frac{1}{(s-\alpha)^{n}} .
$$

Elle est donc vraie pour tout $n=1,2, \ldots$.

La formule (9) permet d'évaluer, par décomposition en fractions simples, tout opérateur rationnel en $s$

$$
\frac{\alpha_{m} s^{m}+\ldots+\alpha_{0}}{\beta_{n} s^{n}+\ldots+\beta_{0}} \quad\left(\alpha_{m}, \beta_{n} \neq 0\right) .
$$

Exemples:

$$
\begin{array}{r}
1^{0} \quad \frac{1}{(s-\alpha)^{2}+\beta^{2}}=\frac{1}{2 \beta i}\left(\frac{1}{s-\alpha-\beta i}-\frac{1}{s-\alpha+\beta i}\right)= \\
=\frac{1}{2 \beta i}\left\{e^{(\alpha+\beta i) t}-e^{(\alpha-\beta i) t}\right\}=\left\{\frac{1}{\beta} e^{\alpha t} \sin \beta t\right\} \\
2^{0} \quad \frac{s-\alpha}{(s-\alpha)^{2}+\beta^{2}}=\frac{1}{2}\left(\frac{1}{s-\alpha-\beta i}+\frac{1}{s-\alpha+\beta i}\right)= \\
=\frac{1}{2}\left\{e^{(\alpha+\beta i) t}+e^{(\alpha-\beta i) t}\right\}=\left\{e^{\alpha t} \cos \beta t\right\} \\
\frac{12}{3^{0}}=\frac{1}{s+2}-\frac{s-1}{(s-1)^{2}+3}+\frac{3}{(s-1)^{2}+3}= \\
=\left\{e^{-2 t}-e^{t} \cos \sqrt{3} t+\sqrt{3} e^{t} \sin \sqrt{3} t\right\}
\end{array}
$$

Si l'on veut éviter l'introduction des nombres complexes dans les calculs, il peut être parfois utile de se servir de la formule suivante (qui se démontre par induction):

$\frac{1}{\left[(s-\alpha)^{2}+\beta^{2}\right]^{n}}=\left\{\frac{e^{\alpha t}}{\left(2 \beta^{2}\right)^{n-1}}\left[A_{n}\left(\beta^{2} t^{2}\right) \cdot \frac{1}{\beta} \sin \beta t-B_{n}\left(\beta^{2} t^{2}\right) \cdot t \cos \beta t\right]\right\}$

où $A_{n}$ et $B_{n}$ sont des polynômes définis par reccurence comme suit:

$$
\begin{aligned}
& A_{1}(x)=1, \quad A_{2}(x)=1, \quad B_{1}(x)=0, \quad B_{2}(x)=1, \\
& A_{n+1}(x)=\frac{2 n-1}{n} A_{n}(x)-\frac{x}{n(n-1)} A_{n-1}(x), \\
& B_{n+1}(x)=\frac{2 n-1}{n} B_{n}(x)-\frac{x}{n(n-1)} B_{n-1}(x) \quad(n=2,5, \ldots) .
\end{aligned}
$$

7. Résolution des équations différentielles linéaires aux coefficients constants. Soit

$$
x^{(n)}+a_{1} x^{(n-1)}+\ldots+a_{n} x=f
$$

une équation différentielle aux coefficients constants $\alpha_{1}, \ldots, \alpha_{n}$, où $f$ est une fonction donnée, continue pour $t \geqslant 0$. On cherche une intégrale $x$ qui satisfasse aux conditions initiales:

$$
x(0)=\gamma_{0}, \quad x^{\prime}(0)=\gamma_{1}, \quad \ldots, \quad x^{(n-1)}(0)=\gamma_{n-1} .
$$

On a en vertu de (8)

$$
s^{n} x+\alpha_{1} s^{n-1} x+\ldots+\alpha_{n} x=\beta_{0} s^{n-1}+\ldots+\beta_{n-1}+f,
$$

où $\beta_{1}=\alpha_{0} \gamma_{i}+\ldots+\alpha_{i} \gamma_{0} \quad(i=0,1, \ldots, n-1)$, et par conséquent

$$
x=\frac{\beta_{0} s^{n-1}+\ldots+\beta_{n-1}}{s^{n}+\alpha_{1} s^{n-1}+\ldots+\alpha_{n}}+\frac{1}{s^{n}+\alpha_{1} s^{n-1}+\ldots+\alpha_{n}} f .
$$

Cette méthode de résolution est bien connue dans le calcul opératoire; nous nous bornerons donc à en donner un seul exemple très simple.

On a à chercher l'intégrale de l'équation

$$
x^{\prime}-x=f, \quad \text { où } f=\left\{(2 t-1) e^{t^{2}}\right\} .
$$

telle que l'on ait $x(0)=0$. On a dans ce cas

$$
s x-x=f
$$

Studia Mathematica. T. XI. 
d'où

$$
x=\frac{1}{s-1} f=\left\{e^{t}\right\}\left\{(2 t-1) e^{t z}\right\}=\left\{\int_{0}^{t} e^{t-\tau}(2 \tau-1) e^{\tau^{2}} d \tau\right\}=\left\{e^{t^{2}}-e^{t}\right\} .
$$

Remarquons que cet exemple n'est pas résoluble par la transformation de Laplace, car l'intégrale $\int_{0}^{\infty} e^{-s t}(2 t-1) e^{t 2} d t$ est divergente.

De manière tout à fait analogue, on peut traiter les systèmes d'équations aux coefficients constants; ces systèmes conduisent à ceux d'équations algébriques que l'on peut résoudre au moyen des déterminants.

\section{Calcul infinitésimal des opérateurs.}

§ 8. Convergence forte des suites de fonctions. Étant donnée une suite $a_{1}, a_{2}, \ldots a_{n}, \ldots$ de fonctions de $C$, nous dirons qu'elle converge fortement vers la fonction a $\epsilon C$ lorsqu'elle converge uniformément dans tout intervalle fermé $0 \leqslant t \leqslant t_{0}$; nous écrirons dans ce cas $\lim a_{n}=a$.

La limite ainsi définie jouit des trois propriétés suivantes:

$1^{0}$ elle est unique, c'est-à-dire: si $\lim a_{n}=a$ et $\lim a_{n}=b$, on a $a=b$;

$2^{0}$ si $a_{n}=a$ pour $n=1,2, \ldots$, on a $\lim a_{n}=a$;

$3^{0}$ si les suites de fonctions $a_{n}$ et $b_{n}$ convergent fortement vers $a$ et $b$ respectivement, chacune des suites $a_{n}+b_{n}, a_{n}-b_{n}$ et $a_{n} b_{n}{ }^{11}$ ) converge fortement et l'on a

$\lim \left(a_{n}+b_{n}\right)=a+b, \quad \lim \left(a_{n}-b_{n}\right)=a-b, \quad \lim a_{n} b_{n}=a b$.

Les démonstrations des propriétés $1^{0}$ et $2^{0}$ et des égalités pour la somme et la différence des suites sont triviales. Pour établir la dernière égalité par exemple, remarquons que les suites des $a_{n}$ et des $b_{n}$ étant fortement convergentes par hypothèse, il existe, pour tout $t_{0}>0$, une suite numérique $\varepsilon_{n}>0$ convergente vers zéro et telle que $\left|a_{n}(t)-a(t)\right|<\varepsilon_{n}$ et $\left|b_{n}(t)-b(t)\right|<\varepsilon_{n}$ pour $0 \leqslant t \leqslant t_{0}$ et $n=1,2, \ldots$

11) $a_{n} b_{n}$ désignant, bien entendu, le produit de composition. si $M \geqslant|a(t)|$ et $M \geqslant|b(t)|$ pour $0 \leqslant t \leqslant t_{0}$, on a

$$
\begin{gathered}
\left|\int_{0}^{t} a_{n}(t-\tau) b_{n}(\tau) d \tau-\int_{0}^{t} a(t-\tau) b(\tau) d \tau\right|= \\
=\mid \int_{0}^{t} a_{n}(t-\tau)\left[b_{n}(\tau)-b(\tau)\right] d \tau+\int_{0}^{t}\left[a_{n}(t-\tau)-a(t-\tau)\right] b(\tau) d \tau \leqslant \\
\leqslant \int_{0}^{t}\left(M+\varepsilon_{n}\right) \varepsilon_{n} d \tau+\int_{0}^{t} \varepsilon_{n} M d \tau \leqslant\left(2 M \varepsilon_{n}+\varepsilon_{n}^{2}\right) t_{0},
\end{gathered}
$$

d'où la convergence forte de $a_{n} b_{n}$ vers $a b$.

§ 9. Fonctions opératoires et fonctions paramétriques. Soit $E$ un ensemble de nombres réels ou complexes. Toute fonction a $(\lambda)$ qui fait correspondre à tout élément $\lambda_{\epsilon} E$ un opérateur a $\epsilon A$ sera dite fonction opératoire.

En particulier, si les valeurs d'une fonction opératoire se réduisent à des éléments de $C$, elle sera dite fonction paramétrique.

La fonction paramétrique $a(\lambda)$ peut donc être considérée comme fonction de deux variables

$$
a(\lambda)=\{a(\lambda, t)\}
$$

ou bien comme une famille de fonctions de $t$, où $\lambda$ est un paramètre.

Nous laisserons de côté, pour le moment, les fonctions opératoires générales et ne nous occuperons que des fonctions paramétriques.

$\$$ 10. Continuité et dérivabilité des fonctions paramétriques. Nous dirons qu'une fonction paramétrique $a(\lambda)$ définie dans l'ensemble $E$ est continue au point $\lambda_{0} \in E$ lorsque la suite des $a\left(\lambda_{n}\right)$ converge fortement vers $a\left(\lambda_{0}\right)$, quelle que soit la suite des $\lambda_{n} \in E$ qui converge vers $\lambda_{0}$.

On démontre comme d'habitude que la somme, la différence et le produit (c'est-à-dire le produit de composition par rapport à $t$ ) des fonctions paramétriques continues est encore une fonction paramétrique continue. 
Nous dirons que la fonction paramétrique $a(\lambda)$ possède la dérivée (relative à $E$ ) $a^{\prime}\left(\lambda_{0}\right)$ au point $\lambda_{0}$ (qui est un point d'accumulation de $E$ ) lorsque la suite des fonctions

$$
\frac{a\left(\lambda_{n}\right)-a\left(\lambda_{0}\right)}{\lambda_{n}-\lambda_{0}}
$$

converge vers $a^{\prime}\left(\lambda_{0}\right)$ pour toute suite des $\lambda_{n} \neq \lambda_{0}$ (où $\lambda_{n} \in E$ ) qui converge vers $\lambda_{0}$ au sens habituel.

Si une fonction paramétrique a $(\lambda)$ est déribable au point $\lambda_{0} \in E$, elle $y$ est continue.

La dérivée des fonctions paramétriques obéit aux règles usuelles :

$$
\left\{\begin{array}{l}
{[a(\lambda)+b(\lambda)]^{\prime}=a^{\prime}(\lambda)+b^{\prime}(\lambda)} \\
{[a(\lambda)-b(\lambda)]^{\prime}=a^{\prime}(\lambda)-b^{\prime}(\lambda)} \\
{[a(\lambda) b(\lambda)]^{\prime}=a^{\prime}(\lambda) b(\lambda)+a(\lambda) b^{\prime}(\lambda)}
\end{array}\right.
$$

en particulier, si $a(\lambda)=a$ ne dépend pas de $\lambda$, on a

$$
[a b(\lambda)]^{\prime}=a b^{\prime}(\lambda) .
$$

De plus, si $\varphi(\lambda)$ est une fonction numérique, dérivable au sens habituel, on a

$$
[a(p(\lambda))]^{\prime}=a^{\prime}(p(\lambda)) p^{\prime}(\lambda) .
$$

Toutes les démonstrations ne s'appuient que sur les propriétés $1^{0}-3^{0}$ du $\S 8$ et sont d'ailleurs les mêmes que dans le calcul différentiel ordinaire.

§ 11. Fonctions paramétriques dans un intervalle. Soit maintenant $a(\lambda)$ une fonction paramétrique définie dans un intervalle fermé $\alpha \leqslant \lambda \leqslant \beta$; on peut donc la considérer (voir $\$ 9$ ) comme une fonction de deux variables réelles $a(\lambda)=\{a(\lambda, t)\}$ définie dans la demi-bande $D$ :

$$
a \leqslant \lambda \leqslant \beta, \quad 0 \leqslant t<\infty .
$$

Pour que la fonction paramétrique a $(\lambda)$ soit continue dans l'intervalle $\alpha \leqslant \lambda \leqslant \beta$, il faut et il suffit que la fonction de deux variables $\{a(\lambda, t)\}$ soit continue au sens habituel dans $D$.

Nécessité. Soit $P_{0}\left(\lambda_{0}, t_{0}\right)$ un point quelconque de $D$; considérons une suite de points $P_{n}\left(\lambda_{n}, t_{n}\right)$ de $D$ convergente vers $P_{0}$.
On a

$a\left(\lambda_{n}, t_{n}\right)-a\left(\lambda_{0}, t_{0}\right)=\left[a\left(\lambda_{n}, t_{n}\right)-a\left(\lambda_{0}, t_{n}\right)\right]-\left[a\left(\lambda_{0}, t_{n}\right)-a\left(\lambda_{0}, t_{0}\right)\right]$.

La première des différences entre crochets tend vers 0 , car la fonction paramétrique est supposée continue; la seconde tend vers zéro, car la fonction $\left\{a\left(\lambda_{0}, t\right)\right\}$ est continue en $t$.

Suffisance. Si la fonction $\{a(\lambda, t)\}$ est continue dans $D$, elle l'est uniformément dans tout rectangle partiel fini $D_{0}$ :

$$
a \leqslant \lambda \leqslant \beta, \quad 0 \leqslant t \leqslant t_{0} .
$$

Donc, si $a_{n} \leqslant \lambda_{n} \leqslant \beta$ et $\lambda_{n}$ converge rers $\lambda_{0}$, la suite des fonctions $\left\{a\left(\lambda_{n}, t\right)\right\}$ tend uniformément vers $\left\{a\left(\lambda_{0}, t\right)\right\}$ pour $0 \leqslant t \leqslant t_{0}$, d'où la continuité de la fonction paramétrique $a(\lambda)$.

Si une fonction paramétrique a $(\lambda)$ définie dans l'intervalle $\alpha \leqslant \lambda \leqslant \beta \quad y$ est déribable (c'est-à-dire possède la dérivée dans tout point de cet intervalle), la fonction de deux variables $\{a(\lambda, t)\}$ possède la dérivée partielle $\left\{\frac{\partial}{\partial \lambda} a(\lambda, t)\right\}$ dans tout point de la demibande $D$ et l'on a

$$
a^{\prime}(\lambda)=\left\{\frac{\partial}{\partial \lambda} a(\lambda, t)\right\}
$$

On a en effet, quel que soit le point $P_{0}\left(\lambda_{0}, t\right)$ de la demi-bande $D$,

$$
\frac{a\left(\lambda_{n}\right)-a\left(\lambda_{0}\right)}{\lambda_{n}-\lambda_{0}}=\left\{\frac{a\left(\lambda_{n}, t_{0}\right)-a\left(\lambda_{0}, t_{0}\right)}{\lambda_{n}-\lambda_{0}}\right\}
$$

pour $\lambda_{n} \neq \lambda_{0}\left(\alpha \leqslant \lambda_{n} \leqslant \beta\right)$.

L'exemple suivant montre que la réciproque n'est pas vraie, c'est-à-dire que l'existence de la dérivée partielle $\left\{\frac{\partial}{\partial \lambda} a(\lambda, t)\right\}$ n'implique pas celle de la dérivée $a^{\prime}(\lambda)$. Soit

$$
a(\lambda, t)=\frac{\lambda(\lambda-t)^{2}}{\lambda^{2}+t^{2}} \quad \text { pour } \lambda, t>0 \text { et } a(0,0)=0 .
$$

La dérivée partielle par rapport à $t$ existe évidemment en tout point du demi-plan $t \geqslant 0$. Cependant la dérivée $a^{\prime}(\lambda)$ n'existe pas pour $\lambda=0$, car

$$
\frac{a\left(\lambda_{n}\right)-a(0)}{\lambda_{n}}=\left\{\frac{\lambda(\lambda-t)^{2}}{\lambda^{2}+t^{2}}\right\} \quad\left(\lambda_{n} \neq 0\right),
$$


et on voit que la dernière suite ne converge uniformément daus aucun intervalle $0 \leqslant t \leqslant t_{0}$.

On a cependant le théorème :

Si la dérivée partielle $\left\{\frac{\partial}{\partial \lambda} a(\lambda, t)\right\}$ est continue dans la demibande $D$, la fonction paramétrique a $(\lambda)$ est dérinable dans l'interoalle $\alpha \leqslant \lambda \leqslant \beta$ et l'on a (13).

Posons, en effet, $b(\lambda, t)=\frac{\partial}{\partial \lambda} a(\lambda, t)$; alors

$$
\frac{a\left(\lambda_{n}\right)-a\left(\lambda_{0}\right)}{\lambda_{n}-\lambda_{0}}=\left\{b\left(\xi_{n}(t), t\right)\right\} \quad \text { pour } \lambda \neq \lambda_{11}, a \leqslant \lambda_{01} \text { et } \lambda_{n} \beta,
$$

où la valeur $\xi_{n}(t)$ est comprise entre $\lambda_{0}$ et $\lambda_{n}$.

Le corollaire suivant est important pour les applications:

Si la dérivée $a^{\prime}(\lambda)$ existe et est nulle dans l'intervalle $\alpha \leqslant \lambda \leqslant \beta$, la fonction paramétrique $a(\lambda)$ est constante par rapport à $\lambda$, c'est-à-dire que $a(\lambda)=a=\{a(t)\}$.

La démonstration resulte immédiatement de deux théorèmes précédents.

\$ 12. Intégrale d'une fonction paramétrique. Nous dirons qu'une fonction paramétrique $a(\lambda)$ est sommable dans l'intervalle $a \leqslant \lambda \leqslant \beta$, lorsque la fonction de deux variables $\{a(\lambda, t)\}$, continue par rapport à $t$, est sommable par rapport à $\lambda$ quel que soit $t \geqslant 0$, et que l'on a en outre

$$
|a(\lambda, t)| \leqslant p(\lambda) c(t) \quad \text { pour } \quad a \leqslant \lambda \leqslant \beta \text { et } t \geqslant 0
$$

où $\varphi(\lambda)$ est une fonction sommable dans l'intervalle $\alpha \leqslant \lambda<\beta$ et $c(t)$ est une fonction continue pour $t \geqslant 0$.

Posons par définition

$$
\int_{a}^{\beta} a(\lambda) d \lambda=\left\{\int_{\alpha}^{\beta} a(\lambda, t) d \lambda\right\} .
$$

L'intégrale $\int_{\alpha}^{\beta} a(\lambda, t) d \lambda$ ainsi définie est une fonction continue de $t$, ce qui résulte du théorème de Lebesciue sur l'intégration des suites.
Les propriétés suivantes de l'intégrale résultent aussitôt de la définition:

$$
\begin{gathered}
\int_{\alpha}^{\beta} a(\lambda) d \lambda+\int_{\beta}^{\gamma} a(\lambda) d \lambda=\int_{\alpha}^{\gamma} a(\lambda) d \lambda, \\
\int_{\alpha}^{\beta}[a(\lambda) \pm b(\lambda)] d \lambda=\int_{\alpha}^{\beta} a(\lambda) d \lambda \pm \int_{\alpha}^{\beta} b(\lambda) d \lambda, \\
\int_{\alpha}^{\beta} a(\lambda) d \lambda=\int_{\alpha+\gamma^{\prime}}^{\beta+\gamma} a(\lambda-\gamma) d \lambda=\frac{1}{\gamma} \int_{\alpha=\prime}^{\beta+\gamma} a\left(\frac{\lambda}{\gamma}\right) d \lambda \quad(\gamma \neq 0) .
\end{gathered}
$$

On roit facilement aussi que

$$
\int_{i}^{b} c a(\lambda) d \hat{\lambda}=\left.c\right|_{i} ^{i^{3}} a(\lambda) d \lambda .
$$

quelle que soit la fonction $c$ de $C$, ce qui résulte de l'identité

$$
\int_{i}^{3} \sum_{i=1}^{n} c\left(\frac{n-i}{n} t\right) a\left(\lambda,{ }_{n}^{i} t\right) d \lambda=\frac{1}{n} \sum_{i=1}^{n} c\left(\frac{n-i}{n} t\right) \int_{i=}^{\beta} a\left(\lambda, \frac{i}{n} t\right) d \lambda_{n}
$$

en faisant $n$ tendre vers l'infini.

\$ 13. Dérivées des fonctions opératoires générales. Nous dirons qu'une fonction opératoire $a(\lambda)$ définie dans un ensemble $E$ possède la dérioée $a^{\prime}\left(\lambda_{0}\right)$ au point $\lambda_{0}$ (qui est un point d'accumulation de $E$ ) lorsqu'il existe un élément non nul $c \in C$ tel que $c a(\lambda)$ est une fonction paramétrique dans un entourage de $\lambda_{0}$ et possède la dérivée $c a^{\prime}\left(\lambda_{0}\right)$ au point $\lambda_{11}$.

Les dérivées des fonctions opératoires obéissent aux mêmes lois (10), (11) et (12) du $\S 11$ que les dérivées des fonctions paramétriques. Les démonstrations sont triviales.

Nous dirons qu'une fonction opératoire $a(\lambda)$, définie dans un intervalle fermé $J$, est régulièrement déribable dans $J$, lorsqu'il existe un élément $k \epsilon C$, universel pour l'intervalle tout entier et tel que $k a(\lambda)$ est une fonction paramétrique dérivable dans $J$.

La somme, la différence et le produit de deux fonctions opératoires régulièrement dérivables sont encore régulièrement dérivables.

Si une fonction opératoire $a(\lambda)$ est régulièrement dérivable dans un intervalle fermé et sa dérivée y est nulle, la fonction $a(\lambda)$ se réduit à une constante (voir $\$ 11$ ). 
Les démonstrations se ramènent trivialement à celles pour les fonctions paramétriques.

Si $\varphi(\lambda)$ est une fonction numérique, on peut l'interpréter aussi comme une fonction opératoire qui fait correspondre des opérateurs numériques aux nombres $\lambda$ (voir $\S 4$ ). On peut donc parler de la dérivée au sens usuel et de la dérivée au sens opératoire. Or, il est facile. de voir que les deux dérivées se confondent; de plus, toute fonction opératoire $p(\lambda)$ qui est dérivable dans un intervalle $J$ y est régulièrement dérivable. Cela résulte aussitôt de la formule $\ln (\lambda)=\{\varphi(\lambda)\}$.

$\S$ 14. Fonetions exponentielles. Étant donné un opérateur $m$ désignons par

la fonction opératoire qui

$1^{0}$ est régulièrement dérivable dans tout intervalle fini $\alpha \leqslant \lambda \leqslant \beta$;

$2^{0}$ satisfait à l'équation différentielle

$$
x^{\prime}(\lambda)=\operatorname{rox}(\lambda)
$$

$3^{0}$ a la valeur 1 pour $\lambda=0$.

On ne sait tout d'abord rien sur l'existence et l'unicité d'une telle fonction. Or, on a les théorèmes suivants:

I. Pour qu'il existe une fonction satisfaisant aux conditions $1^{0}-3^{0}$, il faut et il suffit qu'il existe une fonction paramétrique $p(\lambda)$ définie dans un intervalle $\alpha \leqslant \lambda \leqslant \beta$, non identiquement nulle et satisfaisant a l'équation (14).

II. S'il existe une fonction opératoire satisfaisant aux conditions $1^{0}-3^{0}$, elle est unique.

La nécessité de la condition I est évidente. Pour démontrer qu'elle est suffisante, nous allons nous appuyer sur le lemme:

Si deux fonctions paramétriques $p(\lambda)$ et $q(\lambda)$ satisfont dans l'intervalle $a \leqslant \lambda \leqslant \beta$ à l'équation

$$
a x^{\prime}(\lambda)=b x(\lambda) \quad(a, b \in C ; a \neq 0)
$$

et si l'on a $p\left(\lambda_{0}\right)=q\left(\lambda_{0}\right)$ dans un point $\lambda_{0}$ de cet interpalle, on a identiquement $p(\lambda)=q(\lambda)$ dans l'intervalle $\alpha \leqslant \lambda \leqslant \beta$ tout entier ${ }^{12}$ ).

12) J. G.-Mikusiński [20].
Admettons qu'il existe une fonction paramétrique $p(\lambda)$ qui satisfait à (14) dans l'intervalle $\alpha \leqslant \lambda \leqslant \beta$ et que $p\left(\lambda_{0}\right) \neq 0$ pour un certain point $\lambda_{0}$ de cet intervalle. On peut supposer, sans restreindre la généralité, que $\alpha=v_{0} \gamma$ et $\beta=\left(\nu_{0}+1\right) \gamma$, où $\nu_{0}$ est un entier et $\gamma$ est un nombre positif. En écrivant $m=\frac{b}{a}$, où $a, b \in C$ et $a \neq 0$, on $a$

$$
a p^{\prime}(\lambda)=b p(\lambda)
$$

pour $v_{0} \gamma \leqslant \lambda \leqslant\left(\nu_{0}+1\right) \gamma$; or, si la fonction $p(\lambda)$ était nulle dans un point quelconque de l'intervalle $v_{0} \gamma \leqslant \lambda \leqslant\left(v_{0}+1\right) \gamma$, elle le serait, en vertu du lemme, dans l'intervalle tout entier. On a donc

$$
p(\lambda) \neq 0 \quad \text { pour } v_{0} \gamma \leqslant \lambda \leqslant\left(v_{0}+1\right) \gamma^{\prime} .
$$

En posant

$$
P(\lambda)=p\left(v_{0} \gamma\right)^{\nu-v_{0}-1} \cdot p(\lambda-v \gamma)
$$

pour $\left(v_{0}+v\right) \gamma \leqslant \lambda \leqslant\left(v_{0}+v+1\right)$ et $\nu=0, \pm 1, \ldots$, on vérifie facilement que la fonction opératoire $P(\lambda)$ se trouve définie pour tout $\lambda$ réel et satisfait aux conditions $1^{0}-3^{\circ}$.

Pour démontrer le théorème II, supposons qu'il y ait deux fonctions $P_{1}(\lambda)$ et $P_{2}(\lambda)$ satisfaisant aux conditions $1^{0}-3^{0}$. Fixons arbitrairement un intervalle $a \leqslant \lambda \leqslant \beta$ renfermant le point $\lambda_{0}=0$ à l'intérieur et supposons que les produits

$$
k_{1} P_{1}(\lambda) \text { et } k_{2} P_{2}(\lambda) \text {, }
$$

où $k_{1}, k_{2}$ sont des éléments non nuls de $C$, soient des fonctions paramétriques dans cet intervalle. Les deux produits

$$
k_{1} k_{2} P_{1}(\lambda) \quad \text { et } \quad k_{1} k_{2} P_{2}(\lambda)
$$

satisfont alors à l'équation (15) avec la même condition initiale au point $\lambda=0$. En vertu du lemme, on a donc $k_{1} k_{2} P(\lambda)=k_{1} k_{2} P(\lambda)$ et, par suite, $P_{1}(\lambda)=P_{2}(\lambda)$ dans l'intervalle en question, c. q. f. d.

Les fonctions opératoires exponentielles jouissent des propriétés analogues à celles de la fonction exponentielle ordinaire. On a la formule générale

$$
e^{m \lambda} \cdot e^{m \mu}=e^{i(\lambda+\mu)}
$$

pour tous $\lambda$ et $\mu$ réels. 
En effet, en posant pour un $\mu$

$$
Q(\lambda)=e^{i n \lambda} \cdot e^{m \mu}-e^{m(\lambda+\mu)},
$$

on vérifie facilement que $Q^{\prime}(\lambda)=0$, d'où $Q(\lambda)=Q(0)=0$.

S'il existe des fonctions $e^{m_{1} \lambda}$ et $e^{m_{2} \lambda}$, il existe aussi la fonction $e^{\left(m_{1}+m_{2}\right) \lambda}$ et

$$
e^{m_{1} \lambda} \cdot e^{m_{2} \lambda}=e^{\left(m_{1}+n_{2}\right) \lambda}
$$

Pour le montrer, il suffit de remarquer que le produit $e^{m_{1} \lambda} \cdot e^{m_{2} \lambda}$ satisfait à l'équation différentielle

$$
x^{\prime}(\lambda)=\left(w_{1}+w_{2}\right) x(\lambda) \text {. }
$$

§ 15. Opérateurs de translation. On vérifie sans peine que la fonction paramétrique $p(\lambda)$, définie par la formule

$$
p(\lambda, t)=\left\{\begin{array}{cc}
0 & \text { pour } 0 \leqslant t \leqslant \lambda, \\
1 / 2(t-\lambda)^{2} & \text { pour } 0 \leqslant \lambda \leqslant t,
\end{array}\right.
$$

satisfait pour $\lambda \geqslant 0$ à l'équation différentielle

$$
x^{\prime}(\lambda)=-s x(\lambda)
$$

comme $p(0)=\left\{\begin{array}{l}t^{2} \\ 2 !\end{array}\right\}=l^{3}, \quad$ on a

$$
e^{-s \lambda}= \begin{cases}s^{3} p(\lambda) & \text { pour } \lambda>0 \\ \frac{l^{3}}{p(-\lambda)} & \text { pour } \lambda<0\end{cases}
$$

Si $\lambda$ est fixé positif et $a=\{a(t)\}$ est une fonction continue ou sommable, le produit $e^{-s i}$ a est une fonction $b=\{b(t)\}$ telle que

$$
b(t)=\left\{\begin{array}{cl}
0 & \text { pour } 0 \leqslant t<\lambda, \\
a(t-\lambda) & \text { pour } t \geqslant 0 .
\end{array}\right.
$$

Moins précisément: la multiplication par $e^{-s \lambda}(\lambda=0)$ est la translation $d u$ diagramme de $a=\{a(t)\}$ de longueur $\lambda$ dans la direction positive de l'axe des $t$.

On a en effet

$$
\begin{aligned}
p(\lambda) \cdot a & =\left\{1 / 2 \int_{0}^{1-\lambda}(t-\tau-\lambda)^{2} a(\tau) d \tau\right\}=\left\{1 / 2 \int_{i}^{t}(t-\tau)^{2} a(\tau-\lambda) d\right\}= \\
& \left.=\int_{1 / 2} \int_{0}^{t}(t-\tau)^{2} b(\tau) d \tau\right\}=l^{8} b .
\end{aligned}
$$

L'opérateur $e^{-s i}$ sera dit opérateur de translation.

Remarquons encore que

$$
l e^{-\lambda s}=\{q(\lambda, t)\} \quad \text { pour } \lambda \geqslant 0,
$$

où

$$
q(\lambda, t)= \begin{cases}0 & \text { pour } 0 \leqslant t<\lambda \\ 1 & \text { pour } 0 \leqslant \lambda<t .\end{cases}
$$

\$ 16. Opérateur intégral d'ordre arbitraire. L'opérateur intégral $l$ (voir $\$ 2$ ) satisfait pour tout $n$ naturel à la formule

$$
l^{n}=\left\{\frac{t^{n-1}}{(n-1) !}\right\}
$$

qui se démontre facilement par induction. Cette formule se prête à la généralisation suivante:

$$
l^{\lambda}=\left\{\frac{t^{\lambda-1}}{\Gamma(\lambda)}\right\}
$$

$(\lambda>0)$

où $\Gamma$ désigne la fonction gamma d'Euler.

Le symbole $l^{2}$ se laisse interpréter comme une fonction paramétrique. On peut vérifier que

$$
\left(l^{2}\right)^{\prime}=(s\{\ln t\}+\gamma) l^{2} \quad(\gamma=\text { constante d'Euler })
$$

$l^{i}$ est donc une fonction exponentielle que l'on peut écrire dans la forme

$$
l^{\lambda}=e^{\gamma \lambda} \cdot e^{s\{l n t\} \lambda}
$$

où $e^{\gamma \hat{\pi}}$ est la fonction exponentielle ordinaire et $e^{s\{\ln t\} \bar{\lambda}}$ satisfait à l'équation différentielle

$$
x^{\prime}(\lambda)=s\{\ln t\} x(\lambda) .
$$

D'après le théorème général sur les fonctions exponentielles (voir $\$ 14$ ), la fonction $l^{2}$ est définie aussi pour les valeurs négatives de $\lambda$ et satisfait à l'égalité

$$
l^{2} \cdot l^{\mu}=l^{i+\mu}
$$

pour tous les $\lambda$ et $\mu$ réels. Pour les valeurs positives de $\lambda$ et de $\mu$ la dernière formule se laisse aussi déduire directement au moyen 
de la relation connue entre les intégrales $T$ et $B$ d'Euler. Réciproquement, la voie inverse peut servir à démontrer cette relation.

$\$ 12$. Fonction exponentielle parabolique. Posons pour $\lambda$ et $t$ positifs

$$
F(\lambda, t)=1 / 2 \lambda \pi^{-1 / 2} t^{-3 / 2} \exp \left(-\frac{\lambda^{2}}{4 t}\right)
$$

alors

$$
l^{1 / 2}\{F(\lambda, t)\}=\left\{\frac{\lambda}{2 \pi} \int_{0}^{t}(t-\tau)^{-1 / 2} \tau^{-3 / 2} \exp \left(-\frac{\lambda^{2}}{4 \tau}\right) d \tau\right\}
$$

et, après lă substitution $\frac{\lambda^{2}}{4 \tau}=\frac{\lambda^{2}}{4 t}+\sigma^{2}$,

$$
l^{1 / 2}\{F(\lambda, t)\}=\left\{\frac{2}{\pi \sqrt{t}} \exp \left(-\frac{\lambda^{2}}{4 t}\right) \int_{0}^{\infty} e^{-\sigma^{2}} d \sigma\right\}=\left\{\frac{1}{\sqrt{\pi} t} \exp \left(-\frac{\lambda^{2}}{4 t}\right)\right\} .
$$

On a ensuite

$$
l^{3 / 2} F(\lambda)=\left\{\int_{0}^{t} \frac{1}{\sqrt{\pi \tau}} \exp \left(-\frac{\lambda^{2}}{4 \tau}\right) d \tau\right\} .
$$

Or, la dérivée partielle

$$
\frac{\partial}{\partial \lambda} \int_{0}^{t} \frac{1}{\sqrt{\pi \tau}} \exp \left(-\frac{\lambda^{2}}{4 \tau}\right) d \tau=-\int_{0}^{t} 1 / 2 \lambda \pi^{-1 / 2} \tau^{-3 / 2} \exp \left(-\frac{\lambda^{2}}{4 \tau}\right) d \tau
$$

est continue pour $t \geqslant 0$ et $\lambda>0$; donc $l^{\beta / 2} F^{\prime}(\lambda)=-l F(\lambda)$, d'où (18)

$$
F^{\prime}(\lambda)=-\sqrt{s} F(\lambda) \quad \text { pour } \lambda>0,
$$

où $\sqrt{s}=l^{-1 / 2}$. La fonction opératoire $F(\lambda)$ se laisse prolonger pour tout $\lambda$ réel de manière qu'elle satisfasse à (18). On a en particulier

donc

$$
F(0)=l^{-3 / 2}\left\{\int_{0}^{t} \frac{d \tau}{\sqrt{\pi \tau}}\right\}=l^{-3 / 2}\left\{\frac{t^{1 / 2}}{\Gamma(3 / 2)}\right\}=1 ;
$$

$$
F(\lambda)=e^{-\sqrt{s} \lambda} \text {. }
$$

Pour les valeurs positifs de $\lambda$, la fonction exponentielle $e^{-1 / \lambda}$ se réduit à la fonction paramétrique

$$
e^{-1 \bar{s} \lambda}=\left\{1 / 2 \lambda \pi^{-1 / 2} t^{-3 / 2} \exp \left(-\frac{\lambda^{2}}{4 t}\right)\right\}
$$

qui joue un rôle fondamental dans la théorie de l'équation parabolique.

§ 18. Intégrale d'une fonction opératoire. Nous dirons qu'une fonction opératoire $a(\lambda)$ est intégrable dans l'intervalle $\alpha \leqslant \lambda \leqslant \beta$. lorsque le produit $k a(\lambda)$ est, pour un $k \epsilon C$ convenablement choisi, une fonction paramétrique intégrable dans cet intervalle.

Posons par définition

$$
\int_{\alpha}^{\beta} a(\lambda) d(\lambda)=\frac{1}{k} \int_{\alpha}^{\beta} k a(\lambda) d(\lambda)
$$

Il est presque immédiat que les théorèmes du $\$ 12$ subsistent pour fonctions opératoires générales.

Tout comme c'était le cas de dérivation (voir $\$ 13$ ), l'intégration (ordinaire) d'une fonction numérique et celle (au sens qui vient d'être précisé) de la fonction opératoire correspondante dont les valeurs sont donc des opérateurs numériques - se confondent. Cette isomorphie par rapport à l'intégration s'explique aussitôt par la formule $l \varphi(\lambda)=\{\varphi(\lambda)\}$.

Il est intéressant que si $\varphi(\lambda)$ est une fonction intégrable dans l'intervalle $0 \leqslant \alpha \leqslant t \leqslant \beta$, on a

(19) $\int_{\alpha}^{\beta} e^{-s \lambda} \varphi(\lambda) d \lambda=\{f(t)\}, \quad$ où $f(t)=\left\{\begin{array}{cl}\varphi(t) & \text { pour } a \leqslant t \leqslant \beta \\ 0 & \text { ailleurs. }\end{array}\right.$

On a en effet, en posant (17),

$$
l \int_{a}^{\beta} e^{-s \lambda} \varphi(\lambda) d \lambda=\left\{\int_{0}^{\beta} q(\lambda, t) f(\lambda) d \lambda\right\}=\left\{\int_{0}^{t} f(\lambda) d \lambda\right\}=l\{f(\boldsymbol{t})\} .
$$

\$ 19. Relation avec la transformation de Laplace. Si la fonction numérique $p(\lambda)$ est intégrable dans tout intervalle $0 \leqslant t \leqslant t_{0}$, l'intégrale

$$
\int_{0}^{\beta} e^{-s \lambda} \varphi(\lambda) d \lambda
$$

converge, d'après la formule (19), uniformément vers $\{\varphi(t)\}$ dans tout intervalle $0 \leqslant t \leqslant t_{0}$ lorsque $\beta \rightarrow \infty$. On a donc la formule 


$$
\int_{0}^{\infty} e^{-s \lambda} \gamma(\lambda) d \lambda=\{\varphi(t)\} .
$$

Considérons s, pour l'instant, comme une variable complexe. Alors la formule (20) établit une transformation qui fait correspondre certaines fonctions analytiques à des fonctions $\varphi(\lambda)$ pour lesquelles l'intégrale converge et qui est dite transformation de Laplace.

De cette manière, la transformation de Laplace peut être introduite dans le calcul opératoire comme instrument accessoire. Remarquons toutefois que cet instrument n'est plus applicable si l'intégrale (20) diverge.

Par contre, en revenant à l'intexprétation opératoire, l'intégrale (20) a toujours un sens, quelle que soit la fonction $p(\lambda)$ intégrable dans les intervalles finis $0 \leqslant t \leqslant t_{0}$. Par exemple:

$$
\int_{0}^{\infty} e^{-s \lambda+\lambda^{2}} d \lambda=\left\{e^{t^{2}}\right\}
$$

§ 20. Suites d'opérateurs. Nous dirons qu'une suite d'opérateurs $a_{n}$ conperge vers l'opérateur $a$, lorsqu'il existe un élément non nul $k \in C$ tel que $k a_{n} \in C(n=1,2, \ldots), k a \epsilon C$, et que la suite des $k a_{n}$ converge fortement vers $k a$ (voir $\$ 8$ ); nous poserons par définition

$$
\lim a_{n}=\frac{\lim k a_{n}}{k} .
$$

Toute suite des $a_{n} \in C$ qui converge fortement est évidemment convergente au sens qui vient d'être défini; la convergence des opérateurs est ainsi une généralisation de la convergence forte. Il peut arriver qu'une suite des $a_{n} \epsilon C$ converge en tant qu'une suite d'opérateurs sans qu'elle converge fortement. La suite $\{\cos n t\}$, par exemple, converge vers zéro, car on a pour $k=l$

$$
l\{\cos n t\}=\left\{\frac{1}{n} \sin n t\right\} \rightarrow 0 .
$$

On peut aussi vérifier sans peine que chacune des suites $\left\{n e^{-n t}\right\}, \quad\left\{n^{2} t e^{-n t}\right\}, \quad\{n \sin n t\}, \quad\left\{n-n^{2} t+\left|n-n^{2} t\right|\right\}, \quad\left\{\frac{1}{n} t^{1-1 / n}\right\}$ converge vers l'opérateur 1 et que l'on a

$$
\lim \left\{n^{2} \cos n t\right\}=s, \quad \lim \left\{n^{3} \sin n t+n^{3} t \cos n t\right\}=2 s^{2} .
$$

Pour établir ces formules, il suffit de multiplier les suites qui précèdent par $l^{*}$, l'exposant $x$ étant convenablement choisi. Pour la suite $\left\{n^{2} \cos n t\right\}$, par exemple, on a en posant $x=5$,

$$
l^{3}\left\{n^{2} \cos n t\right\}=\left\{t-\frac{1}{n} \sin n t\right\} \rightarrow\{t\}=l^{2},
$$

d'où $\lim \left\{n^{2} \cos n t\right\}=s$.

Notons que la limite définie ici pour les opérateurs obéit aux lois $1^{0}-3^{0}$ du $\$ 8$. Les démonstrations sont triviales.

§ 21. Séries d'opérateurs et séries de puissances. On écrira

$$
L=\sum_{n=0}^{\infty} a_{n}=a_{0}+a_{1}+a_{2}+\ldots
$$

lorsque $L=\lim \left(a_{0}+\ldots+a_{n}\right)$. Les séries les plus importantes pour les applications sont celles de puissances de la forme

$$
\Phi(\lambda k)=a_{0}+a_{1} \lambda k+a_{2} \lambda^{2} k^{2}+\ldots .
$$

où $a_{0}, a_{1}, \ldots$ et $\lambda$ sont des nombres.

Si le rayon de convergence de la série numérique

$$
\Phi(\lambda)=a_{0}+a_{1} \lambda+a_{2} \lambda^{2}+\ldots
$$

n'est pas nul et $k \epsilon C$, la série $\Phi(\lambda k)$ conoerge pour tout $\lambda$ complexe; on a en outre

$$
[\Phi(\lambda k)]^{\prime}=\alpha_{1} k+2 \alpha_{2} k^{2}+\ldots
$$

et la dernière série converge encore pour tout $\lambda$ complexe.

La première partie de ce théorème résulte aussitôt du lemme suivant:

Si le rayon de convergence de la série numérique

$$
\text { - } \Psi(\lambda)=a_{1} \lambda+a_{2} \lambda^{2}+\ldots
$$

n'est pas nul et $k \epsilon C$, la série

$$
\Psi(\lambda k)=\alpha_{1} \lambda k+a_{2} \lambda^{2} k^{2}+\ldots
$$

converge fortement. 
Pour démontrer le lemme, fixons arbitrairement un intervalle fini $0 \leqslant t \leqslant t_{0}$ et désignons par $M$ un nombre pour lequel on a $k(t) \leqslant M$ dans cet intervalle. Si

$$
k^{1}(t)=k(t) \quad \text { et } \quad k^{n+1}(t)=\int_{0}^{t} k^{n}(t-\tau) k(\tau) d \tau \quad(n=1,2, \ldots),
$$

on a par induction

$$
\left|k^{n}(t)\right|<M^{n} \frac{t^{n-1}}{(n-1) !} \quad \text { pour } 0<t<t_{11},
$$

d'où la convergence uniforme de la série $\Psi(\lambda k)$ dans l'intervalle $0 \leqslant t \leqslant t_{0}$. Comme cet intervalle est arbitraire, la série $\Psi(\lambda k)$ converge fortement, c. q. f. d.

En vertu de ce lomme, on peut dériver la série $\Psi(\lambda k)$ comme d'habitude:

$$
[\Psi(\lambda k)]^{\prime}=a_{1} k+2 \alpha_{2} k^{2}+\ldots ;
$$

il suffit, en effet, de remarquer que la dérivée partielle

$$
\frac{\partial}{\partial \lambda}\left[\alpha_{1} \lambda k(t)+\alpha_{2} \lambda^{2} k^{2}(t)+\ldots\right]
$$

est une fonction continue (voir $\S 11$ ). Or, cela entraine cléjà l'égalité (21) et la seconde partie du théorème.

En particulier, si

$$
\Phi(\lambda k)=1+\frac{\lambda k}{1 !}+\frac{\lambda^{2} k^{2}}{2 !}+\ldots
$$

on a

$$
[\Phi(\lambda k)]^{\prime}=k+\frac{\lambda k^{2}}{1 !}+\frac{\lambda^{2} k^{2}}{2 !}+\ldots=k \Phi(\lambda k) .
$$

On a donc, conformément à la définition des fonctions opératoires exponentielles (voir $\$ 14$ ),

$$
e^{\lambda k}=1+\frac{\lambda k}{1 !}+\frac{\lambda^{2} k^{2}}{2 !}+\ldots
$$

Un autre cas important est celui de la série

$$
\Phi(\lambda k)=1+\lambda k+\lambda^{2} k^{2}+\ldots ;
$$

on vérifie aussitôt que $1+\lambda k \Phi(\lambda k)=\Phi(\lambda k)$, d'où $(1-\lambda k) \Phi(\lambda k)=1$

$$
\frac{1}{1-\lambda k}=1+\lambda k+\lambda^{2} k^{2}+\ldots,
$$

quel que soit le nombre complexe $\lambda$ et la fonction $k \in C$.
$\S$ 22. Séries de translations. Posons pour $\lambda_{0}>0$ fixé arbitrairement

$$
a=e^{-s \lambda_{0}}
$$

Il est facile de voir que la série

$$
a_{0}+a_{1} a+a_{2} a^{2}+\ldots
$$

est convergente, quelle que soit la suite de nombres complexes $a_{0}, \alpha_{1}, \alpha_{2}, \ldots$ En effet, si $k_{\epsilon} C$, le produit $a^{n} k$ est nul dans l'intervalle $0 \leqslant t \leqslant n \lambda$ (voir $\$ 15$ ), d'où la convergence forte de la série $a_{0} k+\alpha_{1} a k+\alpha_{2} a^{2} k+\ldots$ et, par conséquent, la convergence de la série (22). On roit aussi que

$$
\frac{1}{1-\lambda a}=1+\lambda a+\lambda^{2} a^{2}+\ldots
$$

Lopérateur $\frac{1}{1-\lambda a}$ joue un rôle important dans la théorie de l'équation hyperbolique. Pour illustrer l'influence qu'il exerce comme facteur sur les fonctions multipliées, nous donnons ci-dessous les diagrammes des fonctions $l$ et $l^{2}$, en même temps que des produits $l \frac{1}{1-a}$ et $l^{2} \frac{1}{1+2 a}$ :
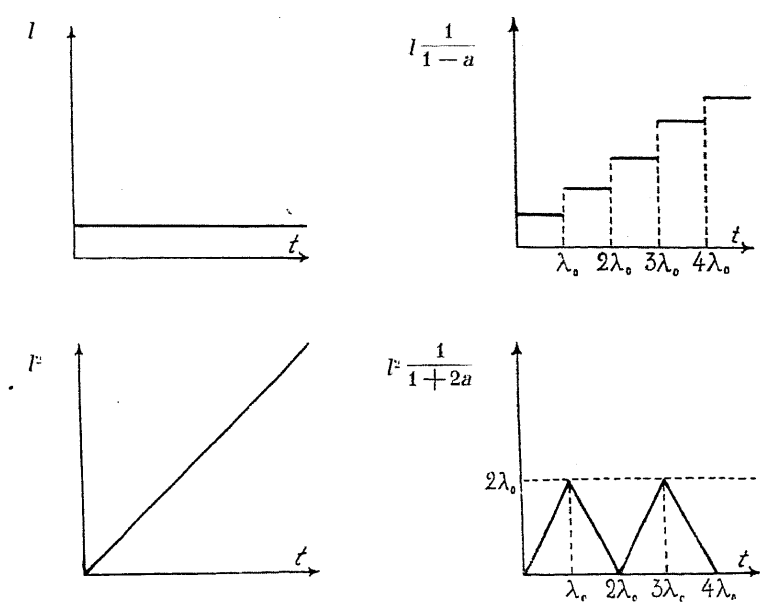

Studia Mathematica. T. XI. 
La forme de ces diagrammes s'explique aussitôt par la formule (23) et par le théorème d'après lequel la multiplication par a revient à la translation du diagramme de longueur $n \lambda_{0}$ (voir $\$ 15)$.

§ 23. Application aux équations aux dérivées partielles. Ce mémoire ne concernant en principe que les fondements du calcul opératoire, nous nous bornerons à des exemples très simples.

L'équation hyperbolique

$$
x_{\lambda \lambda}(\lambda, t)=x_{t t}(\lambda, t)
$$

peut s'écrire dans la notation des opérateurs comme suit:

$$
x^{\prime \prime}=s^{2} x-s p(\lambda)-\eta(\lambda),
$$

où $\varphi(\lambda)=x(\lambda, 0)$ et $\psi(\lambda)=x_{t}(\lambda, 0)$.

Occupons nous d'abord de l'équation homogène

$$
x^{\prime \prime}=s^{2} x .
$$

En cherchant les solutions exponentielles de la forme $x=e^{n \pi}$ on est conduit à la condition $r^{2}=s^{2}$, d'où $r= \pm s$. On voit donc que la fonction

$$
x(\lambda)=c_{1} e^{-s \lambda}+c_{2} e^{s \lambda}
$$

satisfait, quels que soient les opérateurs $c_{1}$ et $c_{2}$, à l'équation (26).

$\mathrm{Si}$ l'on cherche une solution pour laquelle $x\left(\lambda_{1}\right)=k_{1}$ et $x\left(\lambda_{2}\right)=k_{2}$, où $k_{1}$ et $k_{2}$ sont des opérateurs donnés arbitrairement on a à résoudre le système d'équations

$$
c_{1} e^{-s \lambda_{1}}+c_{2} e^{s \lambda_{1}}=k_{1}, \quad c_{1} e^{-s \lambda_{2}}+c_{2} e^{s \lambda_{2}}=k_{2} .
$$

On trouve ainsi

$$
c_{1}=\frac{k_{1} e^{s \lambda_{1}}-k_{2} e^{-s \lambda_{2}+2 s \lambda_{1}}}{1-e^{-2 s\left(\lambda_{2}-\lambda_{1}\right)}}, \quad c_{2}=\frac{-k_{1} e^{-2 s \lambda_{2}+s \lambda_{1}}+k_{2} e^{-s \lambda_{2}}}{1-e^{-s\left(\lambda_{2}-\lambda_{1}\right)}}
$$

et l'intégrale peut être évaluée par les développements en séries.

Reprenons maintenant le cas général (25). En posant

$$
x(\lambda)=c_{1}(\lambda) e^{-s \lambda}+c_{2}(\lambda) e^{s} \lambda
$$

et en appliquant la méthode connue de variation des constantes, on trouve l'intégrale particulière

$$
x(\lambda)=\frac{1}{2} \int_{\dot{z}_{0}}^{\lambda}\left[e^{-s(\lambda-x)}-e^{s(\lambda-x)}\right] \cdot[\varphi(x)+l \psi(x)] d x,
$$

qui permet de trouver à son tour la solution générale et de l'ajuster aux conditions initiales ou bien à celles aux limites.

On peut traiter d'une manière tout à fait analogue l'équation parabolique

On a dans ce cas

$$
x_{\lambda \lambda}(\lambda, t)=x_{t}(\lambda, t)
$$

$$
x^{\prime \prime}=s x-a(\lambda) .
$$

L'équation homogène a la solution

$$
x(\hat{\lambda})=c_{1} e^{-l s \lambda}+c_{2} e^{l i \bar{z}},
$$

et on peut établir pour l'équation non homogène une intégrale particulière analogue à (27).

§ 24. Sur l'unicité des solutions. La méthode des opérateurs permet aussi d'établir des théorèmes d'unicité pour les équations partielles. Nous nous bornons ici à quelques exemples simples.

On a d'abord le lemme suivant:

Si deux fonctions paramétriques $p(\lambda)$ et $q(\lambda)$ satisfont dans l'intervalle $\alpha \leqslant \lambda \leqslant \beta$ à l'équation différentielle

$$
a x^{\prime \prime}(\lambda)+b x(\lambda)=c(\lambda)
$$

où $a, b \in C, a \neq 0$ et $c(\lambda)$ est une fonction paramétrique donnée, et si l'on a $p\left(\lambda_{0}\right)=q\left(\lambda_{0}\right)$ et $p^{\prime}\left(\lambda_{0}\right)=q^{\prime}\left(\lambda_{0}\right)$ dans un point $\lambda_{0}$ de cet intervalle, on $y$ a identiquement $p(\lambda)=q(\lambda)$ partout $\left.{ }^{13}\right)$.

On déduit de ce lemme le théorème suivant:

Si la fonction exponentielle $e^{m \lambda}(m \neq 0)$ existe et $x_{0}(\lambda)$ est une solution particulière de l'équation non homogène

$$
x^{\prime \prime}-m^{2} x=c(\lambda)
$$

toute fonction $x(\lambda)$ satisfaisant à cette équation se laisse représenter dans la forme

$$
x(\lambda)=c_{1} e^{-m \lambda}+c_{2} e^{m \lambda}+x_{0}(\lambda),
$$

où $c_{1}$ et $c_{2}$ sont des opérateurs convenablement choisis.

\footnotetext{
13) J. G.-Mikusiński [20].
} 
En vertu du lemme, il suffit de remarquer que le système d'équations

$$
\begin{gathered}
x\left(\lambda_{0}\right)=c_{1} e^{-w \lambda_{0}}+c_{2} e^{m \lambda_{0}}+x_{0}\left(\lambda_{0}\right), \\
x^{\prime}\left(\lambda_{0}\right)=-c_{1} m e^{-m \lambda_{0}}+c_{2} m e^{m \lambda_{0}}+x_{0}^{\prime}\left(\lambda_{0}\right)
\end{gathered}
$$

est univoquement résoluble en $c_{1}$ et $c_{2}$, ce qui est sûrement le cas, car

$$
\left|\begin{array}{cc}
e^{-m \lambda_{0}} & e^{m \lambda_{0}} \\
-m e^{-m \lambda_{0}} & m e^{m \lambda_{0}}
\end{array}\right|=2 m \neq 0
$$

Si, de plus, $e^{m \lambda} \neq 1$ pour $\lambda \neq 0$, la solution de l'équation (22) est déterminée uniooquement dans l'interballe $\alpha \leqslant \lambda \leqslant \beta$ par les valeurs limites $\boldsymbol{x}(\alpha)$ et $\boldsymbol{x}(\beta)$.

En effet, en vertu du théorème précédent, il suffit maintenant de remarquer que le système des équations

$$
\begin{aligned}
& x(\alpha)=c_{1} e^{-m \alpha}+c_{2} e^{r a \alpha}+x_{0}(\alpha), \\
& x(\beta)=c_{1} e^{-m \beta}+c_{2} e^{m \beta}+x_{0}(\beta)
\end{aligned}
$$

est univoquement résoluble, ce qui est vrai, car

$$
\left|\begin{array}{ll}
e^{-m c \alpha} & e^{w c} \\
e^{-m \beta} & e^{m \beta}
\end{array}\right|=e^{-m(\beta-\alpha)}\left[e^{2 w(\beta-\alpha)}-1\right] \neq 0 .
$$

Voici deux corollaires directs:

L'intégrale $x(\lambda, t)$ de l'équation hyperbolique (24) est déterminée univoquement dans le domaine $D$ :

par ses baleurs:

$$
\alpha \leqslant \lambda \leqslant \beta, \quad 0 \leqslant t<\infty
$$

$x(\lambda, 0)$ et $x_{t}(\lambda, 0)$ pour $\alpha \leqslant \lambda \leqslant \beta, x(x, t)$ et $x(\beta, t)$ pour $0 \leqslant t<\infty$.

Pareillement, l'intégrale $x(\lambda, t)$ de l'équation parabolique (28) est déterminée uninoquement dans $D$ par ses valeurs:

$x(\lambda, 0)$ pour $a \leqslant \lambda \leqslant \beta, \quad x(\alpha, t)$ et $x(\beta, t)$ pour $0 \leqslant t<\infty$.

Voici encore deux théorèmes:

Si la solution $x(\lambda, t)$ de l'équation hyperbolique (24) est telle que $\boldsymbol{x}(\lambda, 0)=x_{t}(\lambda, 0)=0$ pour $a \leqslant \lambda \leqslant \beta$ et $x\left(\gamma_{1}, t\right)=x\left(\gamma_{2}, t\right)=x\left(\gamma_{3}, t\right)$ pour $0 \leqslant t<\infty$, où $\gamma_{1}, \gamma_{2}$ et $\gamma_{3}$ sont trois points distincts de l'interballe $\alpha \leqslant \lambda \leqslant \beta$, on a identiquement $x(\lambda, t)=0$ dans $D$.
Pareillement, si la solution $x(\lambda, t)$ de l'équation parabolique (28) est telle que $x(\lambda, 0)=0$ pour $a \leqslant \lambda \leqslant \beta$ et $x\left(\gamma_{1}, t\right)=x\left(\gamma_{2}, t\right)=x\left(\gamma_{3}, t\right)$ pour $0 \leqslant t<\infty$, on a identiquement $x(\lambda, t)=$ dans $D$.

En effet, on est alors conduit au système d'équations

$$
c_{1} e^{-m ; 1}+c_{2} e^{m \gamma_{1}}=c_{1} e^{-m \gamma^{2}}+c_{2} e^{m ; \mu}=c_{1} e^{-m ; / 3}+c_{2} e^{m \% 3},
$$

d'où $c_{1}=c_{2}=0$

Les résultats de ce paragraphe ne peuvent pas être obtenus moyennant la transformation de Laplace, à moins de restreindre la classe des fonctions admissibles.

\section{Publications citées.}

[1\} Berg, E. J., Heaviside's operational calculus, Electrical Engineering Text, $2^{\text {nd }}$ edition, Harvard University 1936.

[2] Carslaw, H. and Jaeger, J., Operational methods in Applied mathematics, New York 1941.

[3] Carson, J. R., Electric circuit theory and operational calculus, New York 1926.

[4] Churchill, R. V., Modern Operational Mathematics in Engineering, New York 1944.

[5] Crum, M. M., On the resultant of two functions, The Quarterly Journal of Mathematics, Oxford Series 12, No 46, 1941, p. 108-111.

[6] Dahr, K., A course of integrational and operational calculus with applications to problems of physics and electrotechnics, Stockholm 1935.

[7] Ditkin, V. A., Operacionnoe isčisienie, Uspiehi Matiematičeskih Nauk II, 6 (22), 1947, p. 72-158.

[8] Doetsch, G., Theorie und Anrendung der Laplace-Transformation, Berlin 1957.

[9] Drobot, S., et Mikusiński, J. G.-, Sur l'unicité des solutions de quelques équations différentielles dans les espaces abstraits (I), ce volume, p. 38-40.

[10] Dufresnoy, J., Sur le produit de composition de deux fonctions, Comptes rendus de l'Académie des Sciences 225, Paris 1947, p. 857-859.

[11] - Autour du théorème de Phragmén-Lindelöf, Bulletin des Sciences Mathématiques 72,1948 , p. 17-22.

[12] Efros, A. i Danilevskij, A., Operacionnoe isčislenie i konturnyie integraly, Charkov 1937.

[13] H e a visi d e, O., Electromagnetic theory, London 1899.

[14] Humbert, P., Le calcul symbolique, Actualités scientifiques et industrielles, Paris 1934. 
[15] Jeffreys, H., Operational methods in mathematical physics, $2^{\text {nd }}$ edition, Cambridge Tracts, 23, 1931.

[16] Lurie, A., Operacionnoe isčislenie $v$ priloženiah $k$ zadačam mehaniki, Moscou 1938.

[17] Mc Lachlan, N. W., Complex Variable and Operational Calculus mith technical applications, Cambridge 1939.

[18] - Modern Operational Calculus, London 1948.

[19] Mikusiński, J. G.-, L'anneau algébrique el ses applications dans l'analyse fonctionnelle, I, Annales Universitatis Mariae Curie-Skłodowska, Lublin, Sectio A II (1948), p. 1-48; - II, ibidem, III (1949), p. 1-84.

[20] - Sur l'unicité des solutions de quelques équations différentielles dans les espaces abstraits (I), Aunales de la Société Polonaise de Mathématique 22 (1949), p. 157-160.

[21] - Une nouvelle justification du calcul opératoire, Atti dell'Academia $\mathrm{Na}$ zionale dei Lincei (à paraître).

[22] Titchmarsh, E. C., The zeros of certain integral functions, Proceedings of the London Mathematical Society 25, 1926, p. 283-302.

[23] - Introduction to the theory of Fourier Integrals, Oxford 1937.

[24] Vahlen, K. T., Zeitschrift für angerbandte Mathematik und Mechanik, 13, 1935, p. 283-298.

[25] Wag n e r, K. W., Operalorenrechnung, Berlin 1940.

(Reçu par la Rédaction le 5. 5. 1949)

\section{Weak compactness in Banach spaces}

by

\section{G. SIRVINT $\dagger$ (Leningrad).}

The author was murdered by the Germans during the second world war. The present work was received by the editor in 1941 and has been prepared for print by A. Alexiewicz.

In his monography [4] ${ }^{1}$ ) BANACH calls only little attention to the notion of weak compactness. In recent years, however, the importance of this concept for Functional Analysis has been emphasized in several papers, to mention only its role in the theory of stochastic processes. The present paper ${ }^{2}$ ) deals with a detailed study of the weakly compact subsets of Banach spaces, especially of the separable ones. When considering the compact subsets in Banach spaces, I follow the way indicated by Gelfand [10].

The contents of this paper consist of two parts. In the first part $(\S 1-\S z)$, necessary and sufficient conditions for weak compactness of subsets of separable Banach spaces are given and the connectedness of the weak convergence with the weak compactness are studied. In the second part $(\$ 8-\S 10)$ we consider the general forms of weak compactness in some concrete Banacl spaces and we apply the results to the study of weakly completely continuous operations.

1) The numbers in brackets refer to the bibliography at the end of the present paper (p. 93-94).

2) the principal results of which were announced without proofs in the note [15]. 